\title{
Amyotrophic Lateral Sclerosis Mortality in the United States, 2011-2014
}

\author{
Theodore C. Larson ${ }^{a}$ Wendy Kaye ${ }^{b}$ Paul Mehta ${ }^{a}$ D. Kevin Horton ${ }^{a}$ \\ ${ }^{a}$ Division of Toxicology and Human Health Sciences, Agency for Toxic Substances and Disease Registry, Atlanta, GA, \\ USA; ${ }^{\text {} M c K i n g ~ C o n s u l t i n g ~ C o r p o r a t i o n, ~ A t l a n t a, ~ G A, ~ U S A ~}$
}

\section{Keywords}

Amyotrophic lateral sclerosis · Motor neuron disease .

Mortality

\section{Abstract}

Background: The International Classification of Disease, 10th Revision (ICD-10) did not include a code specific for Amyotrophic lateral sclerosis (ALS) until 2017. Instead, code G12.2 included both ALS and other motor neuron diseases (MND). Our objective was to determine US mortality rates for ALS exclusively by excluding other MND and progressive supranuclear palsy. Methods: All mortality data coded as G12.2 under the pre-2017 rubric were obtained for 2011-2014. Deaths without ALS listed in one of the un-coded cause-ofdeath fields were excluded. ALS death rates per 100,000 persons were age-adjusted to the 2000 US standard population using the direct method. Results: The proportion of excluded records coded G12.2 but not ALS was 0.21 , resulting in 24,328 ALS deaths. The overall age-adjusted mortality rate was $1.70(95 \% \mathrm{Cl} 1.68-1.72)$. The rate among males was 2.09 (95\% Cl 2.05-2.12) and females was 1.37 (95\% Cl 1.35-1.40). The overall rate among whites was 1.84 , blacks 1.03 , and other races 0.70 . For both sexes and all races, the rate increased with age and peaked among 75-79 year-olds. Rates tended to be greater in states at higher latitudes. Conclusions: Previous reports of ALS mortality in the United States showed similar age, sex, and race distributions but with greater ageadjusted mortality rates due to the inclusion of other diseases in the case definition. When using ICD-10 data collected prior to 2017, additional review of multiple-cause of death data is required for the accurate estimation of ALS deaths.

(c) 2018 The Author(s) Published by S. Karger AG, Basel

\section{Introduction}

Amyotrophic lateral sclerosis (ALS) is a rare and progressive neurodegenerative disease involving loss of cortical and lower motor neurons in the spinal cord and brain stem [1]. The clinical features of ALS include signs and symptoms of progressive muscle atrophy, fatigue, and problems with swallowing, which typically lead to respiratory failure and death [1]. The insidiously progressive nature of ALS results in death among most patients within 2-5 years of diagnosis [2]. Although rare with an 
estimated US prevalence of 5.0 cases per 100,000 population [2], ALS exacts an immense burden for patients, caregivers, and society [3].

ALS makes up about $70 \%$ of cases in a disease class known as the motor neuron disease (MND); other main clinical phenotypes of MND are isolated bulbar palsy (4$8 \%$ of cases), progressive muscular atrophy (5\%), and primary lateral sclerosis (1-3\%) [4]. Although ALS is the most common MND, it did not have its own code in the US prior to the 2017 update of the International Classification of Diseases, 10th Revision (ICD-10) rubric. Prior to this update, the ICD-10 code for MND, G12.2, included both ALS and other MND.

The objective for the present analysis was to determine ALS mortality rates for the entire United States for the period 2011-2014, ascertained using ICD-10 code G12.2 as coded using the pre-2017 rubric, but excluding deaths from other MND and other diseases, including progressive supranuclear palsy (G23.1).

\section{Methods}

Multiple-cause mortality data for all deaths involving MND were obtained from the National Center for Health Statistics, Centers for Disease Control and Prevention, for all 50 US states and Washington DC during 2011-2014. This time period was selected to match initial, complete years of data collection conducted by the National ALS Registry, a registry of US cases operated by the Agency for Toxic Substances and Disease Registry [5]. MND-related deaths were defined as the ICD-10 code G12.2. Un-coded causes of death as reported in the medical certification section of the death certificate obtained by National Center for Health Statistics were manually reviewed and records in which ALS was not listed as a cause of death were excluded.

ALS death rates per 100,000 persons were then age-adjusted to the 2000 US standard population using the direct method [6]. Population estimates used in SEER*Stat software [7] were a modification of the annual time series of July 1 county population estimates by age, sex, and race/ethnicity determined by the US Census $\mathrm{Bu}$ reau [8]. Modifications included bridged single-race estimates derived from original, multiple race categories in the 2000 and 2010 Censuses [9] and accounted for known issues in certain counties [8]. The modified county-level population estimates, summed to the state and national levels, were used as denominators in rate calculations [8].

For the rate calculations, ages were grouped in 5-year intervals to 84 and 85 years and older. Corresponding 95\% CIs were calculated as a modification of intervals based on a gamma approximation for rates and an approximation of the $\mathrm{F}$ distribution for rate ratios [10]. Death rates were examined by sex, age, race (black, white, or other), Hispanic origin, year of death, and place of residence at death (state, Census division, and state tier). A map showing the geographic distribution of age-adjusted death rates used the 25 th, 50 th, and 75 th percentiles of state rates as cut points. For

Amyotrophic Lateral Sclerosis Mortality

in the United States, 2011-2014 further rate calculations, state of residence was aggregated into tiers and Census divisions. The northern tier includes states generally north of 41-42 degrees north latitude; the southern tier consists of those states lying south of 37 degrees north latitude; and the middle tier consists of the remaining states. All rates and average annual percent change (APC) in rates were calculated using SEER*Stat software [7].

\section{Results}

There were 30,804 deaths in the United States coded as G12.2 during the study period. Of those deaths, 6,476 (21\%) were excluded from this analysis. Among the excluded decedents, other MND or neurologic disorders frequently appeared as a cause of death: 5,039 with progressive supranuclear palsy; 558 bulbar palsy; 331 primary lateral sclerosis; 80 progressive muscular atrophy; 351 with a nonspecific term for MND; and 117 with some other cause. After exclusions, 24,328 ALS deaths remained for 2011-2014. The majority of ALS decedents were white $(n=22,245 ; 91 \%)$ and deaths among men predominated $(n=13,611 ; 56 \%)$. The age range of the decedents was 14-102 years (4 decedents were younger than 20 years). Age-adjusted rates, expressed per 100,000 population, were stable between years (Table 1) with an annual percentage change that was not statistically significant $(p>0.05)$.

The overall rate for all 24,328 ALS deaths was 1.70 (95\% CI 1.68-1.72); for male decedents, the rate was 2.09 (95\% CI 2.05-2.12); and for female decedents, the rate was 1.37 (95\% CI 1.35-1.40; Table 1). The overall rate ratio for males was 1.52 (95\% CI 1.48-1.56), and a similar ratio between sexes was seen across age groups (Fig. 1).

Overall rate ratios for race were 2.64 for whites and 1.48 for blacks compared with other races (Table 1). The rate ratio for non-Hispanics compared to that of the decedents of Hispanic origin was 1.85 (Table 1). Similar patterns by race and by Hispanic origin were observed across age groups (Figs. 2, 3, respectively). The overall rate for decedents aged $0-49$ years was 0.22 and increased with age through the 70-79 years category (rate $=9.71$ ) and decreased among those aged $\geq 80$ years (Table 1). Figure 2 shows a similar pattern in the age-adjusted rates using 5 -year age categories, reaching a maximum rate in the $70-79$ years category (overall rate in this age category $=$ 10.59; male rate $=12.46$; female rate $=9.12$ ). The APC for rates by sex, race, Hispanic origin, and age was not statistically significant.

The choropleth in Figure 4 visually displays rates by state and suggests states at greater latitudes are associated 
Table 1. Characteristics and age-adjusted rates of ALS-associated deaths in the United States during 2011-2014

\begin{tabular}{|c|c|c|c|c|}
\hline & Number* & $\operatorname{Rate}^{\dagger}(95 \%$ CI $)$ & $\mathrm{RR}^{\ddagger}(95 \% \mathrm{CI})$ & Ratio $p$ value \\
\hline All deaths & 24,328 & $1.70(1.68-1.72)$ & - & \\
\hline 2011 & 5,905 & $1.72(1.67-1.76)$ & 1 & \\
\hline 2012 & 6,149 & $1.74(1.70-1.79)$ & $1.01(0.98-1.05)$ & 0.4850 \\
\hline 2013 & 5,982 & $1.65(1.61-1.69)$ & $0.96(0.93-1.00)$ & 0.0316 \\
\hline Male & 13,611 & $2.09(2.05-2.12)$ & $1.52(1.48-1.56)$ & $<0.0001$ \\
\hline Female & 10,717 & $1.37(1.35-1.40)$ & 1 & \\
\hline \multicolumn{5}{|l|}{ Race } \\
\hline White & 22,245 & $1.84(0.64-0.76)$ & $2.64(2.42-2.89)$ & $<0.0001$ \\
\hline Black & 1,540 & $1.03(0.98-1.09)$ & $1.48(1.34-1.64)$ & $<0.0001$ \\
\hline Other & 543 & $0.70(0.64-0.76)$ & 1 & \\
\hline $0-49$ & 1,761 & $0.22(0.21-0.23)$ & $0.09(0.08-0.09)$ & $<0.0001$ \\
\hline $50-59$ & 4,461 & $2.51(2.44-2.58)$ & 1 & \\
\hline $60-69$ & 7,635 & $5.98(5.84-6.11)$ & $2.38(2.30-2.47)$ & $<0.0001$ \\
\hline $70-79$ & 6,931 & $9.71(9.48-9.94)$ & $3.87(3.73-4.02)$ & $<0.0001$ \\
\hline$\geq 80$ & 3,540 & $7.72(7.47-7.98)$ & $3.08(2.94-3.22)$ & $<0.0001$ \\
\hline \multicolumn{5}{|l|}{ State tier ${ }^{\S}$} \\
\hline Northern & 6,501 & $1.89(1.85-1.94)$ & $1.22(1.18-1.26)$ & $<0.0001$ \\
\hline Middle & 7,912 & $1.77(1.73-1.81)$ & $1.14(1.11-1.17)$ & $<0.0001$ \\
\hline Southern & 6,501 & $1.55(1.52-1.58)$ & 1 & \\
\hline \multicolumn{5}{|l|}{ Census division"l } \\
\hline West North Central & 1,934 & $2.01(1.92-2.10)$ & $1.17(1.10-1.25)$ & $<0.0001$ \\
\hline New England & 1,403 & $1.99(1.89-2.10)$ & $1.16(1.10-1.25)$ & 0.0001 \\
\hline
\end{tabular}

* Number of deaths.

† Average annual mortality rates per 100,000 persons and age-adjusted to the 2000 US standard population.

₹ Rate ratios with $95 \%$ CIs.

$\S$ Northern: AK, CT, ID, MA, ME, MI, MN, MT, ND, NE, NH, NY, RI, SD, VT, WI \& WY. Middle: CO, DC, DE, IA, IL, IN, KS, KY, MD, MO, NJ, NV, OH, PA, UT, VA \& WV. Southern: AL, AR, AZ, CA, FL, GA, HI, LA, MS, NC, NM, OK, SC, TN \& TX.

II West North Central: IA, KS, MN, MO, NE, ND \& SD. New England: CT, ME, MA, NH, RI \& VT. East North Central: IN, IL, MI, OH \& WI. East South Central: AL, KY, MS \& TN. Mountain: AZ, CO, ID, NM, MT, UT, NV \& WY. Pacific: AK, CA, HI, OR \& WA. South Atlantic: DE, DC, FL, GA, MD, NC, SC, VA \& WV. West South Central: AR, LA, OK \& TX. Mid-Atlantic: NJ, NY \& PA. ALS, amyotrophic lateral sclerosis.

with higher mortality rates. The rate for the northern tier of states was 1.89 with a rate ratio of 1.22 compared with the southern tier $(p<0.0001$; Table 1$)$. The confidence intervals for rate and rate ratios at each state tier were non-overlapping (Table 1). Stratifying the state tier re- sults by race showed a similar trend among whites but not among blacks and other races (Table 2). Similarly, the rate ratios among Hispanics did not suggest an association between the state tier and ALS mortality in that demographic (Table 2). Comparing other Census divisions, the 
Fig. 1. ALS-associated average annual death rates in the United States by age group and sex, 2011-2014. ALS, amyotrophic lateral sclerosis.
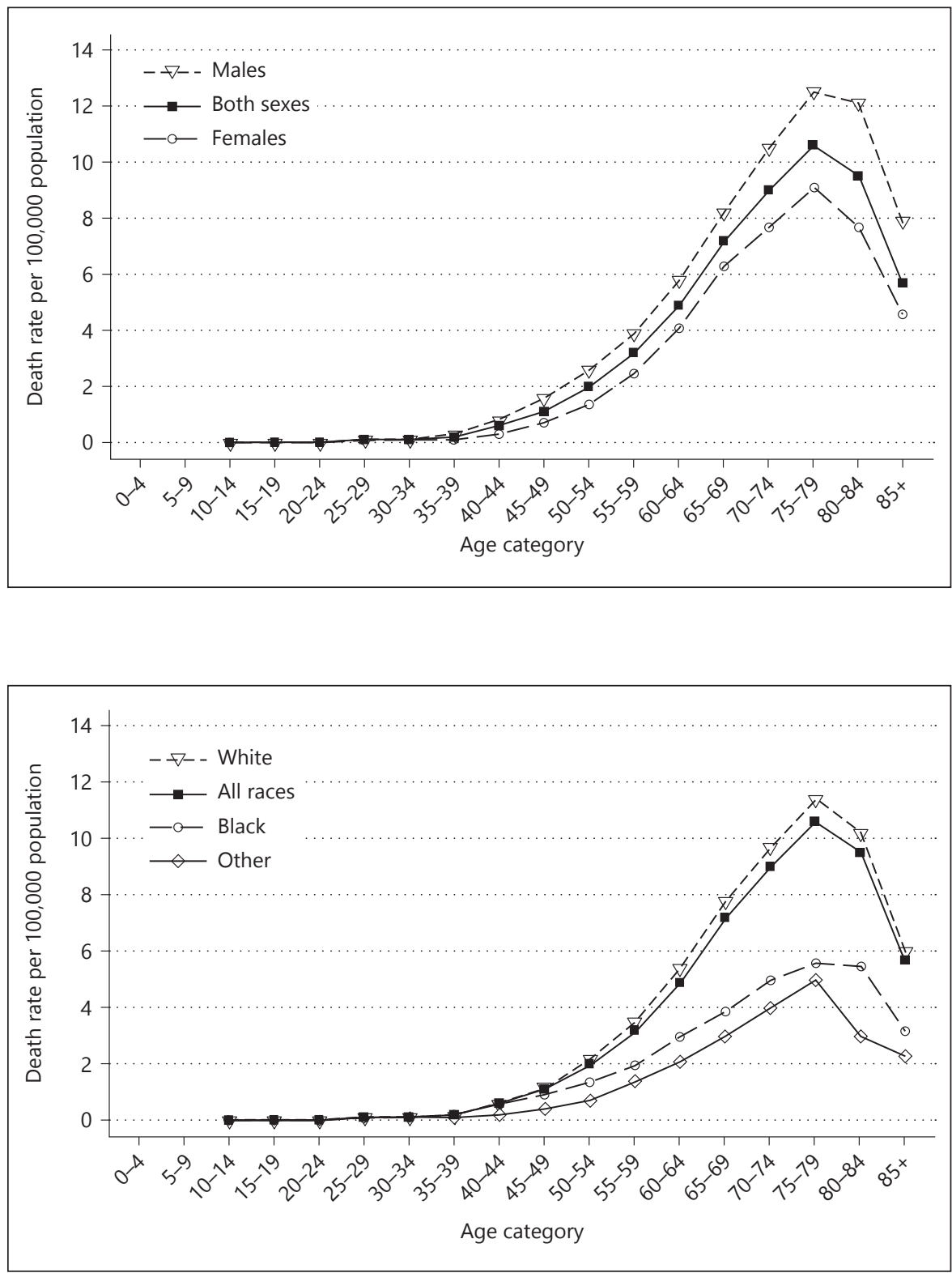

Fig. 2. ALS-associated average annual death rates in the United States by age group and race, 2011-2014. ALS, amyotrophic lateral sclerosis.

\section{Discussion}

We found the ALS mortality rate was 1.70 per 100,000 in the United States. Consistent with the proportion of excluded death records, this rate is about $21 \%$ lower than that found by Mehal et al. [11] in a study that did not exclude other MNDs or progressive supranuclear palsy over the period 1999-2009. In the present study, rates by subpopulations showed elevations among males and whites that increased with age until approximately 70-79 years. These subpopulations also had increased rates in previous studies of ALS mortality in the United 
Fig. 3. ALS-associated average annual death rates in the United States by age group and Hispanic origin, 2011-2014. ALS, amyotrophic lateral sclerosis.
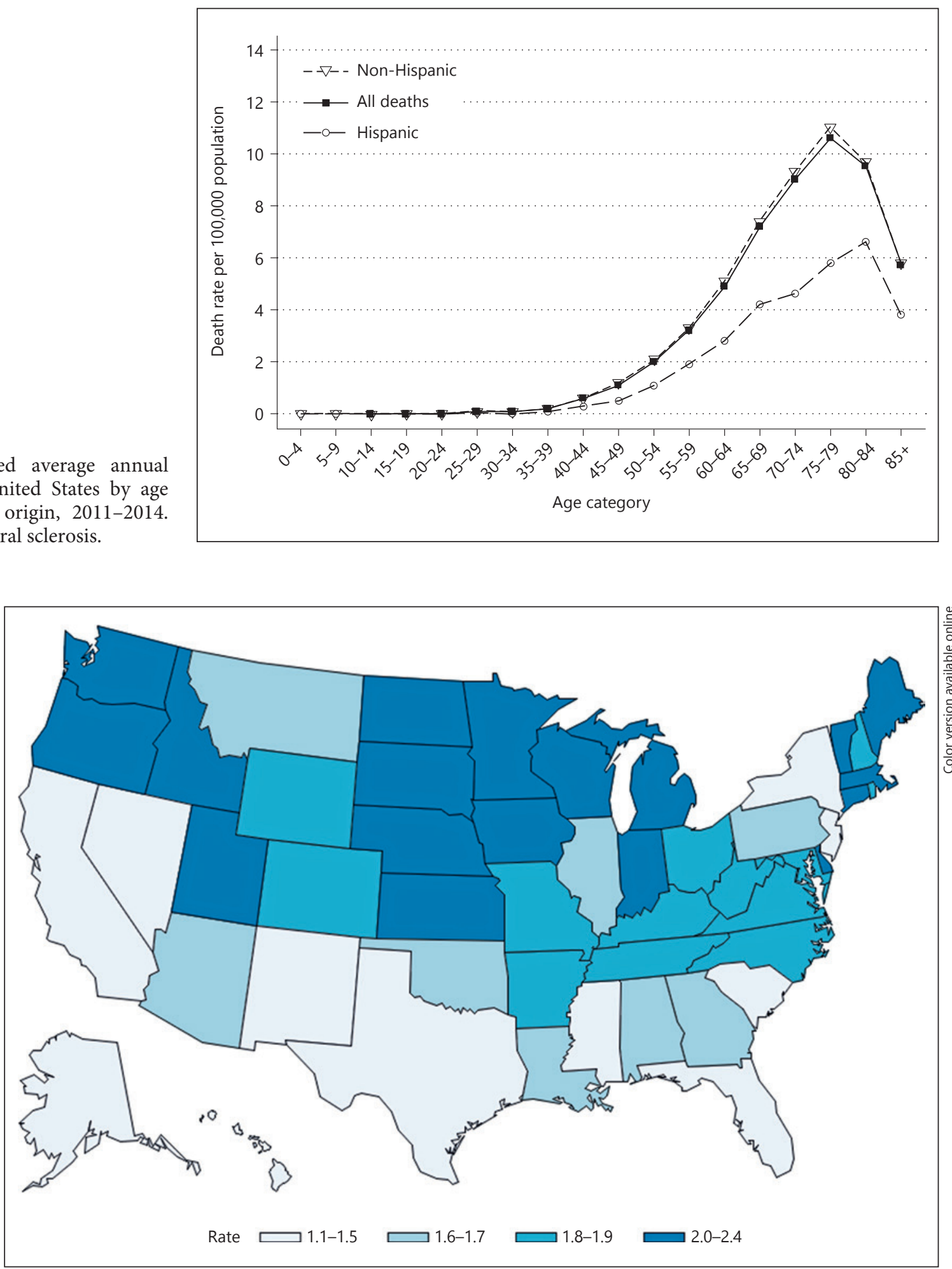

Fig. 4. Geographic distribution of average annual age-adjusted ALS mortality rates per 100,000 population in the United States, 2011-2014. The rate for Washington DC is in the 1.1-1.5 category. 
Table 2. Age-adjusted ALS death rates stratified by race or Hispanic origin and by state tier

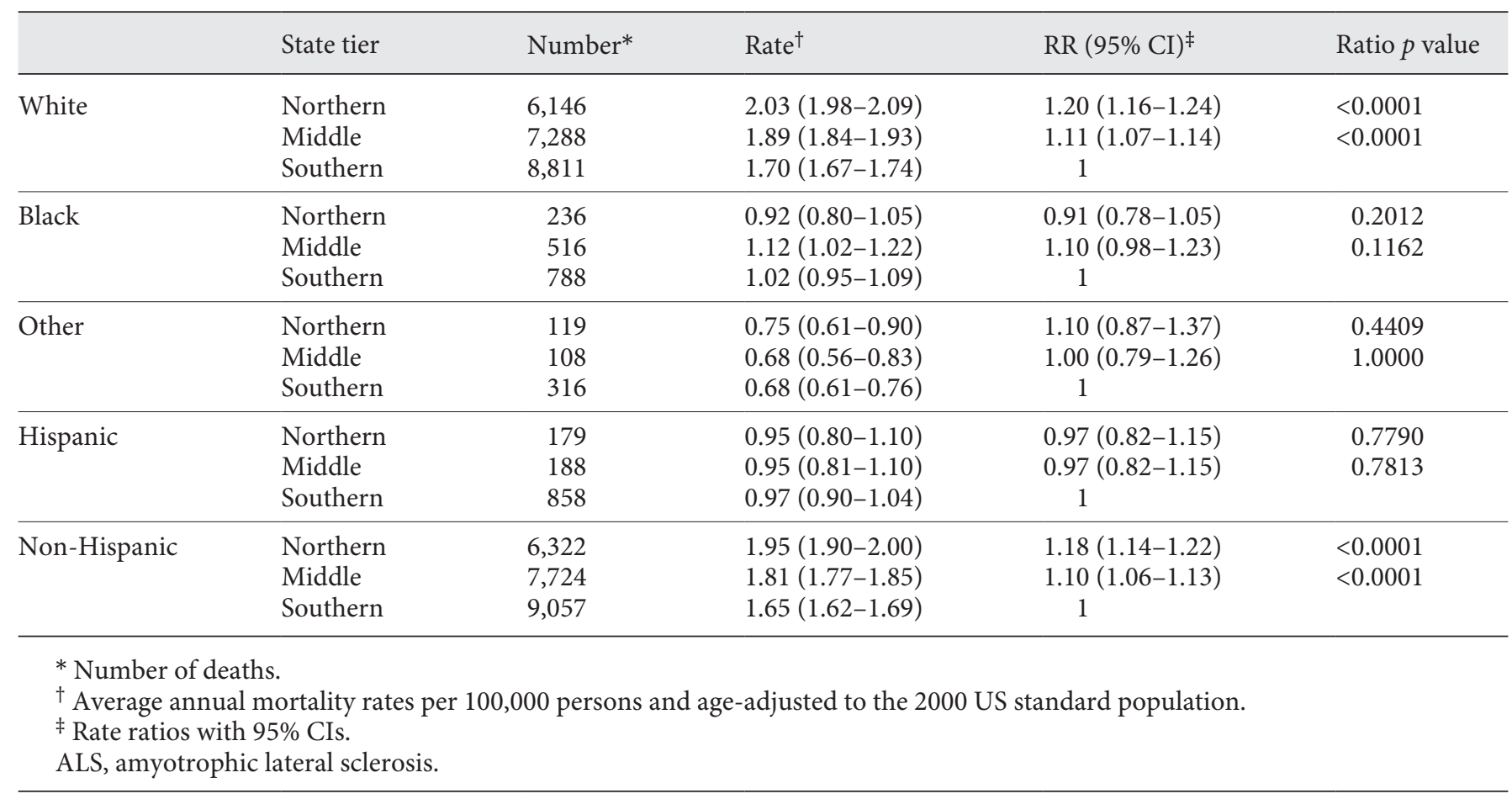

States $[11,12]$. The fact that ALS incidence and prevalence is greater among men has been a consistent finding across studies [13]. This difference between sexes may be due to occupational exposures that historically occurred more commonly among men [14] or a neuroprotective effect of estrogen [15]. ALS incidence has been characterized as increasing with age though age 75 and rapidly declining thereafter [14]. This decline in incidence may be due to difficulties with ascertainment among the elderly. Al-Chalabi and Hardiman note that "older patients are more likely to be referred to nonneurological services, and ALS might be under-recognized in older people because being weak or wasted may be regarded as a normal part of ageing, or may be ignored in someone with multiple medical problems" [13].

Consistent with the race-specific mortality rates that we found, other studies have found lower incidence and mortality rates among non-whites [16, 17]; however, the majority of population-based epidemiologic studies of ALS have been persons of European ancestry [13]. Similarly, other studies have also found lower ALS mortality rates among Hispanics [1, 17]. Lower incidence and mortality among non-whites and His-

Amyotrophic Lateral Sclerosis Mortality

in the United States, 2011-2014 panics may reflect under-ascertainment among nonwhites or a genetic factor predisposing whites to ALS [17].

We found no statistically significant temporal trend in the overall mortality rate, but perhaps this should be expected given that only 4 years of data were analyzed. Still, Mehal et al. [11] found the overall US mortality rate (for all deaths coded G12.2) was stable during the period 1999-2009.

We found evidence of a tendency for states at more northern latitudes to have greater age-adjusted ALS mortality rates than states at more southern latitudes. This is consistent with previous US mortality studies of MND and ALS $[11,12,18]$. A recent review of global ALS epidemiology showed a trend of ALS incidence to increase with higher latitudes among European countries [19]. These regional differences in ALS rates may be due to patient factors, including environmental exposures and genetic predisposition [19]. Our finding of an association between northern latitudes and increased mortality rates among whites only may be related to such factors (e.g., geographic patterns of European immigrant settlement in the United States). An additional study of patient factors, such as access to health care and socioeconomic sta- 
tus, and their relationship to ALS mortality should be considered.

In order to accurately identify ALS, the National ALS Registry submitted a modification request to the committee overseeing the ICD-10 codes in the United States, specifically, the addition of G12.21 for ALS. This change has been made in the 2017 revision and will allow accurate identification of incidence, prevalence, and mortality of ALS in the United States.

This study is limited by the accuracy of death certificates for ALS. However, as the diagnosis of ALS is typically not ambiguous at advanced stages [20], one would expect the rate of false-negative misclassification of ALS as a cause of death to be low. To explore this, we examined mortality data from the Agency for Toxic Substances and Disease Registry's National ALS Registry, 2010-2013. The Registry actively identifies US ALS cases using data from national health services programs (Medicare, Medicaid, and medical assistance programs administered by the Veteran's Administration) and passively via a web portal [5]. Using National Index Data to determine the multiple cause of death for deceased cases, the true positive rate was 0.865 (i.e., among 17,642 registrant deaths, 15,252 had ICD-10 code G12.2 appearing as a cause of death; 95\% CI $0.859-0.870$ ). This suggests that death certificate accuracy for ALS in the United States is good and approxi- mately in the middle of the range of published true positive rates for ALS on death certificates [21]. In addition, when comparing our results to those from other countries, one should be mindful of international differences in ICD-10 rubrics (e.g., ICD-10 Clinical Modification in the United States, ICD-10 Australian Modification, etc.). Differences between rubrics may be seen in the 5th position of specific disease codes [22], which is the position that differentiates between different clinical phenotypes of MND.

In summary, compared with other MND subtypes, ALS is the most malignant with shortest survival [4]. Excluding deaths from other MNDs and progressive supranuclear palsy in data coded using the pre-2017 ICD-10 rubric reduces the total number of deaths attributed to ALS and the adjusted death rate by about $21 \%$. Consistent with other studies, ALS mortality rates with these exclusions were greater among men and whites and generally increased with age. There is evidence of an association between higher latitudes and increased ALS mortality. Although rare, ALS is a devastating disease that imparts large economic impact to patients and society [23]. In addition to ongoing ALS mortality surveillance, the National ALS Registry monitors ALS prevalence in the United States [5]; such Registry data are important for trend analysis and the identification of possible risk factors.

\section{References}

1 Kiernan MC, Vucic S, Cheah BC, Turner MR, Eisen A, Hardiman O, Burrell JR, Zoing MC: Amyotrophic lateral sclerosis. Lancet 2011; 377:942-955.

2 Mehta P, Kaye W, Raymond J, Wu R, Larson T, Punjani R, Heller D, Cohen J, Peters T, Muravov O, Horton K: Prevalence of Amyotrophic Lateral Sclerosis - United States, 2014. MMWR Morb Mortal Wkly Rep 2018; 67:216-218.

3 Galvin M, Corr B, Madden C, Mays I, McQuillan R, Timonen V, Staines A, Hardiman $\mathrm{O}$ : Caregiving in - a mixed methods approach to the study of Burden. BMC Palliat Care 2016;15:81.

4 Dharmadasa T, Henderson RD, Talman PS, Macdonell RA, Mathers S, Schultz DW, Needham M, Zoing M, Vucic S, Kiernan MC: Motor neurone disease: progress and challenges. Med J Aust 2017;206:357-362.

5 Mehta P, Kaye W, Bryan L, Larson T, Copeland T, Wu J, Muravov O, Horton K: Prevalence of Amyotrophic Lateral Sclerosis United States, 2012-2013. MMWR Surveill Summ 2016;65:1-12.
6 Szklo M, Nieto FJ: Epidemiology: Beyond the Basics. Burlington, Jones \& Bartlett Learning, 2014.

7 Surveillance Research Program NCI: SEER*Stat Software, Version 8.3.4. 2017.

8 National Cancer Institute. Surveillance Epidemiology and End Results (SEER) Program: Population Estimates Used in NCI's SEER*Stat Software; Bethesda, MD, National Cancer Institute, 2017. URL: https://seer. cancer.gov/popdata/methods.html (accessed March 28, 2018).

9 Centers for Disease Control and Prevention. National Vital Statistics Program: U.S. Census Populations with Bridged Race Categories, 2017. URL: https://www.cdc.gov/nchs/nvss/ bridged_race.htm (accessed March 28, 2018).

10 Tiwari RC, Clegg LX, Zou Z: Efficient interval estimation for age-adjusted cancer rates. Stat Methods Med Res 2006;15:547-569.

11 Mehal JM, Holman RC, Schonberger LB, Sejvar JJ: Amyotrophic lateral sclerosis/motor neuron disease deaths in the United States, 1999-2009. Amyotroph Lateral Scler Frontotemporal Degener 2013;14:346-352.
12 Sejvar JJ, Holman RC, Bresee JS, Kochanek KD, Schonberger LB: Amyotrophic lateral sclerosis mortality in the United States, 19792001. Neuroepidemiology 2005;25:144-152.

13 Al-Chalabi A, Hardiman O: The epidemiology of ALS: a conspiracy of genes, environment and time. Nat Rev Neurol 2013;9:617628.

14 Logroscino G, Traynor BJ, Hardiman O, Chio' A, Couratier P, Mitchell JD, Swingler RJ, Beghi E: Descriptive epidemiology of amyotrophic lateral sclerosis: new evidence and unsolved issues. J Neurol Neurosurg Psychiatry 2008;79:6-11.

15 de Jong S, Huisman M, Sutedja N, van der Kooi A, de Visser M, Schelhaas J, van der Schouw Y, Veldink J, van den Berg L: Endogenous female reproductive hormones and the risk of amyotrophic lateral sclerosis. J Neurol 2013;260:507-512.

16 Rechtman L, Jordan H, Wagner L, Horton DK, Kaye W: Racial and ethnic differences among amyotrophic lateral sclerosis cases in the United States. Amyotroph Lateral Scler Frontotemporal Degener 2015;16:65-71. 
17 Cronin S, Hardiman O, Traynor BJ: Ethnic variation in the incidence of ALS: a systematic review. Neurology 2007;68:1002-1007.

18 Noonan CW, White MC, Thurman D, Wong LY: Temporal and geographic variation in United States motor neuron disease mortality, 1969-1998. Neurology 2005;64:1215-1221.

19 Chio A, Logroscino G, Traynor BJ, Collins J, Simeone JC, Goldstein LA, White LA: Global epidemiology of amyotrophic lateral sclerosis: a systematic review of the published literature. Neuroepidemiology 2013;41:118-130.
20 Benetar M: Neuromuscular Disease: Evidence and Analysis in Clinical Neurology. Totowa, Humana Press Inc., 2006.

21 Marin B, Couratier P, Preux PM, Logroscino G: Can mortality data be used to estimate amyotrophic lateral sclerosis incidence? Neuroepidemiology 2011;36:29-38.
22 Jette N, Quan H, Hemmelgarn B, Drosler S, Maass C, Moskal L, Paoin W, Sundararajan V, Gao S, Jakob R, Ustun B, Ghali WA: The development, evolution, and modifications of ICD-10: challenges to the international comparability of morbidity data. Med Care 2010; 48:1105-1110.

23 van der Steen, I, van den Berg JP, Buskens E, Lindeman E, van den Berg LH: The costs of amyotrophic lateral sclerosis, according to type of care. Amyotroph Lateral Scler 2009; 10:27-34 